\title{
STUDIES ON THE IN VITRO AND IN VIVO HOST RANGE OF PORCINE EPIDEMIC DIARRHOEA VIRUS
}

\author{
Anna Utiger, ${ }^{1}$ Annekäthi Frei, ${ }^{1}$ Ana Carvajal, ${ }^{2}$ and Mathias Ackermann ${ }^{1}$ \\ ${ }^{1}$ Institute of Virology \\ Vet.- Med. Faculty, University of Zürich \\ Zürich, Switzerland \\ ${ }^{2}$ Dept. de Patologia Animal \\ Fac. Vet., Universidad de Leon \\ Leon, Spain
}

One of the main reasons for the lack of information concerning the biological and molecular properties of porcine epidemic diarrhoea virus (PEDV) has been the difficulty to grow the agent in cell cultures. Interestingly, primary cells as well as cell lines of porcine origin have been found to support poorly, if at all, replication of PEDV. The same was true for cells and cell lines originating from other species such as mouse, bovine, hamster, and humans. Hofmann and Wyler were the first ones to report replication of PEDV in Vero cells. ${ }^{1}$ Later, a Japanese strain of PEDV could be adapted to grow in a slightly broader range of cells. ${ }^{2}$ Interestingly, it was observed that PEDV did not replicate in cell culture unless high amounts of trypsin were present in the culture medium throughout infection. Requirement for trypsin could be explained by two different hypothesis: (i) the receptor attachment protein of the virus had to be cleaved before adsorption or translocation into the cells was possible. Requirement of trypsin to enhance the infectivity in this way is known for many viruses, including corona-, rota-, and influenza viruses. (ii) Presence of trypsin was required to treat surface proteins of the cells, before attachment or translocation or even later events in virus replication were possible. Such treatment is likely to cause detachment of cells from plastic or glass support in conventional cell cultures. Before addressing the above questions, it was necessary to establish cell lines which are resistant to high amounts of trypsin during virus infection.

In order to adapt potential host cells of PEDV to the presence of increasing amounts of trypsin, confluent monolayers of diploid human lung cells (WI-38, ATCC\#CCL75) were subjected to trypsin treatment as follows. The cell culture medium which contained $8 \%$ of fetal calf serum (FCS) was replaced by virus infection medium which was free of FCS but contained $0.3 \%$ tryptose phosphate broth and $2.5 \mu \mathrm{g} / \mathrm{ml}$ of trypsin. ${ }^{3}$ The cells were kept for two hours at $4^{\circ} \mathrm{C}$ and for 24 hours at $37^{\circ} \mathrm{C}$. Free floating and dead cells were then removed. The remaining cells were submerged in fresh cell culture medium containing FCS and left 


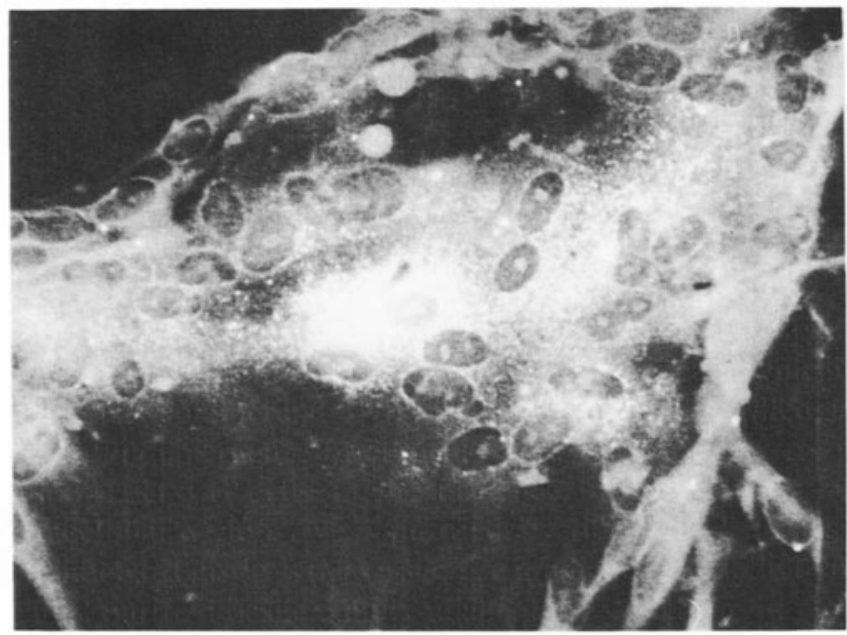

Figure 1. Immunofluorescence of PEDV infected WI-38 cells is shown. Monolayers of WI-38 cells were infected with PEDV (prev1ously passaged already three times in WI-38 cells). 24 hours post infection, the cells were fixed with methanol and incubated consecutively with a monoclonal antibody specific for the N protein of PEDV (mAb2715, kindly supplied by L $\mathrm{Johr}^{4}$ ) and goat antı-mouse FITC

at $37^{\circ} \mathrm{C}$ until the monolayer was restored. Then, the selective treatment was repeated with 5 $\mu \mathrm{g} / \mathrm{ml}$ and thereafter with $10 \mu \mathrm{g} / \mathrm{ml}$ trypsin.

As soon as the WI-38 cells appeared to tolerate $10 \mu \mathrm{g}$ of trypsin for 24 hours, they were infected with our PEDV strain which had been adapted to replicate in Vero cells. ${ }^{1}$ The virus could be passaged in WI-38 cells for at least 5 times. Characteristic syncytium formation was observed from the very first passage on WI-38. With time, the virus obviously adapted to the new cellular system, since more complete and more rapid formation of syncytia was observed. Immunofluorescent assays indicated that all the major structural proteins of PEDV $(\mathrm{S}, \mathrm{M}, \mathrm{N})$ were synthesized in WI-38 infected cells. An example is shown in Fig. 1. Furthermore, coronavirus-like particles purified from the cell culture supernatant could be detected in the electron microscope (not shown).

These observations indicated that the host range of PEDV may be more extended than one has believed until now. It was never established, whether PEDV represented a genuine pig virus or whether it had been introduced to the porcine species from other animals or humans. Therefore, it was of interest to determine if PEDV or a related virus circulated in animal species other than porcines. In order to address this question, sera of humans and of cats were tested for antibodies to PEDV by using the PEDV specific ELISA described by Knuchel et al.. ${ }^{3}$ The details of these studies are reported elsewhere. ${ }^{5}$ Interestingly, 6 out of 165 human sera and 9 out of 47 cat sera reacted positively in the PEDV ELISA. The interpretation of these observations is very tedious because many individuals, humans as well as cats, had also antibodies against other coronaviruses, e.g. human coronavirus $229 \mathrm{E}$ and feline infectious peritonitis virus. Although definitive proof is still lacking, we present for the first time serological evidence that PEDV or a closely related virus may circulate in humans and cats. Our observations are sustained by a report of Have et al. ${ }^{6}$ who have described a PEDV-related virus in mink.

\section{ACKNOWLEDGMENTS}

These studies were supported by the Swiss Federal Institutions BAG and BVet. 


\section{REFERENCES}

1. Hofmann and Wyler, J. Clin. Microbiol., 1988;26, 2235-2239.

2. Kusanagi et al., J. Vet. Med. Sci., 1992;54, 313-318.

3. Knuchel et al., Vet. Microbiol., 1992;32, 117-134.

4. Jöhr, Thesis, Vet. med. Faculty, University of Zürich (1989).

5. Frei, Thesis, Vet. med. Faculty, University of Zürich (in preparation).

6. Have et al., Vet. Microbiol., 1992;31, 1-10. 